\title{
Oui à la protection des enfants et des jeunes contre la publicité pour le tabac
}

\author{
Barbara Weil ${ }^{a}$, Linda Hadorn ${ }^{b}$, Carlos Beat Quinto ${ }^{c}$ \\ ${ }^{a}$ Cheffe de la division Santé publique de la FMH; ${ }^{b}$ Collaboratrice scientifique, division Santé publique de la FMH; ${ }^{c}$ Dr méd., membre du Comité central, \\ responsable du département Santé publique et professions de la santé
}

La grande majorité des fumeuses et des fumeurs commencent à fumer à l'adolescence. Dans ce contexte, la publicité joue un rôle essentiel. Le but de l'initiative populaire «Oui à la protection des enfants et des jeunes contre la publicité pour le tabac» est de protéger les enfants et les jeunes contre la publicité pour le tabac et de leur permettre de grandir sainement.

Les produits du tabac sont les seuls biens de consommation ne pouvant être consommés sans risque pour la santé et susceptibles d'entraîner rapidement une addiction sévère. Le tabagisme est mauvais pour la santé, provoque des maladies graves et génère des coûts indirects importants à la charge de la société. En Suisse, près de 9500 personnes décèdent chaque année des conséquences de la consommation de tabac. Des études montrent aussi que 57 pour cent des fumeurs ont commencé à fumer alors qu'ils étaient encore mineurs. Des études scientifiques prouvent que les jeunes qui sont souvent exposés à la publicité pour le tabac ont tendance à devenir fumeurs.

Environ 3000 jeunes ont été interrogés dans le cadre de l'étude "Le tabagisme chez les 15 à 25 ans en 2016", menée par le Monitorage suisse des addictions. 31,6 pour cent d'entre eux ont déclaré qu'ils fumaient quotidiennement ou occasionnellement, ce qui représente environ six points de pourcentage de plus que dans l'ensemble de la population. La publicité pour le tabac joue un rôle important dans ce contexte.

Contrairement à la plupart des pays européens, la publicité pour les produits du tabac, la promotion des ventes et le parrainage sont omniprésents en Suisse. L'industrie du tabac utilise des stratégies très subtiles pour que la promotion de ses produits soit très visible pour le public cible (les jeunes et les fumeurs) tout en restant relativement discrète pour le reste de la population. Dans le classement $2017 \mathrm{du}$ «Tobacco Control Scale in Europe», la Suisse occupe seulement la $21^{\mathrm{e}}$ place sur la liste de 35 Etats. Par ailleurs, dans le domaine des «Mesures contre la publicité en faveur du tabac», la Suisse n'obtient que 2 points sur 13, ce qui représente le plus mauvais résultat dans cette catégorie.
Le texte de l'initiative «Oui à la protection des enfants et des jeunes contre la publicité pour le tabac» est le suivant:

Initiative populaire fédérale "Oui à la protection des enfants et des jeunes contre la publicité pour le tabac»

La Constitution ${ }^{1}$ est modifiée comme suit:

Art. 41, al.1, let. $g$

1 La Confédération et les cantons s'engagent, en complément de la responsabilité individuelle et de l'initiative privée, à ce que:

g. les enfants et les jeunes soient encouragés à devenir des personnes indépendantes et socialement responsables et soient soutenus dans leur intégration sociale, culturelle et politique et à ce que leur santé soit promue.

\section{Art. 118, al. 2, let. $b$}

2 Elle légifère sur:

b. la lutte contre les maladies transmissibles, les maladies très répandues et les maladies particulièrement dangereuses de l'être humain et des animaux; elle interdit notamment, pour les produits du tabac, toute forme de publicité qui atteint les enfants et les jeunes.

\section{Art. 197, ch. $12^{2}$}

12. Disposition transitoire ad art. 118, al. 2, let. b (Protection de la santé)

L’Assemblée fédérale adopte les dispositions législatives d'exécution dans les trois ans qui suivent l'acceptation de l'art. 118, al. 2, let. b, par le peuple et les cantons. 


\section{Initiative populaire fédérale} «OUI à la protection des enfants et des jeunes contre la publicité pour le tabac》

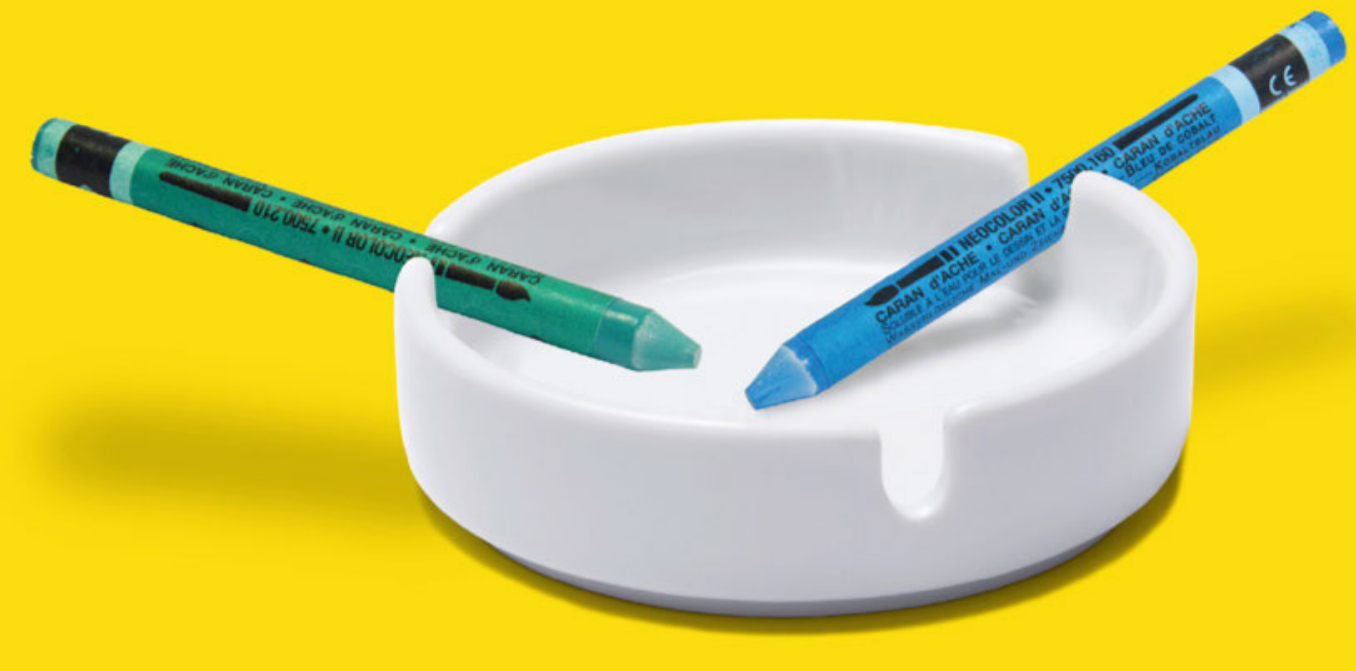

L'initiative est lancée par les grandes organisations suisses de la santé, notamment l'Alliance pour la santé en Suisse, l'Association suisse des droguistes, la FMH, la Ligue suisse contre le cancer, les ligues pulmonaires cantonales, mfe Médecins de famille et de l'enfance Suisse, les pneumologues et les cardiologues, et la
Société suisse des pharmaciens pharmaSuisse. Elle est également soutenue par le Conseil Suisse des Activités de Jeunesse.

\section{Crédit photo}

L'association "Oui à la protection des enfants et des jeunes contre la publicité pour le tabac»
Correspondance:

FMH

Département Santé publique Elfenstrasse 18

Case postale 300

CH-3000 Berne 15

public.health[at]fmh.ch

\section{Participez!}

Si vous souhaitez soutenir l'initiative "Oui à la protection des enfants et des jeunes contre la publicité pour le tabac», demandez à vos amis et à vos connaissances de signer le formulaire de signatures ci-joint.

Vous pouvez télécharger l'argumentaire, les formulaires de signatures et affiches sous www.enfantssanstabac.ch Pour la récolte de signatures, veuillez noter:

- Il est interdit de distribuer des cadeaux ou des gadgets dans le cadre de la récolte de signatures pour une initiative populaire

- Seules les personnes ayant le droit de vote, c'est-à-dire les citoyennes et les citoyens suisses âgés de 18 ans révolus, sont autorisées à signer

- Les noms doivent être inscrits lisiblement à la main et être accompagnés de la signature manuscrite

- Les formulaires qui ne comportent qu'une seule signature sont également valables

- Récolter uniquement les signatures des habitants d'une même commune sur un formulaire

- Les formulaires signés doivent être renvoyés d'ici au 30 septembre 2018 